Gesnerus 70/1 (2013) 7-16

\title{
Un itinéraire de recherche sur l'histoire du corps
}

Georges Vigarello

\section{Summary}

As a pioneer of a new field of research, history of the body, Georges Vigarello goes back over his career as a historian, not so much in search of a hypothetical or ultimate unity of the body, but beyond the original dipersion is rather aiming at relating the existence of coherences and unified logics passing through different fields: the body as an object of thought, of representations and practices. In such a perspective, the history of the body sheds light on a specific era, underlines its culture, while indicating its possible homogeneity.

Keywords: history of the body, representations of the body, models of the body, practices of the body

\section{Résumé}

Figure pionnière d'un nouveau champ de recherche, l'histoire du corps, Georges Vigarello revient sur son parcours d'historien, à la recherche non pas d'une hypothétique unité dernière du corps, mais visant à retracer, au-delà des dispersions premières, l'existence de cohérences, de logiques unifiées traversant des champs différents: le corps comme objet de pensée, de représentations et de pratiques. Dans cette perspective, l'histoire du corps éclaire une époque, souligne sa culture, autant qu'elle en indique la possible homogénéité.

S'entretenir, se soigner, se laver, s'alimenter, se vêtir, s'embellir, jouer..., les «terrains» explorés depuis plusieurs années dans mes textes sont largement différents les uns des autres. L'histoire proposée est, au premier regard,

Georges Vigarello, Ecole des Hautes Etudes en Sciences Sociales, Paris (vigarello@noos.fr). 
plurielle: portant sur le corps, ses transformations, ses apparences, sa sensibilité, elle est d'emblée «diversité». Mes travaux sur le viol par exemple ne semblent guère avoir de rapport avec ceux portant sur la santé ou ceux portant sur la beauté. Les femmes et les hommes qui, dans le temps, inventent le linge, se soignent, se nourrissent, se regardent, s'exercent, s'agressent, se lavent, surveillent le climat, effectuent tous, dans chaque cas, autant de gestes physiques n'ayant guère de correspondances entre eux. L'étude de ces gestes contribue sans doute à l'histoire de la culture corporelle occidentale. Elle ne semble pas, en revanche, faire exister un objet «homogène». Elle ne saurait, tout au moins, convaincre, a priori, de son unité. Chacun de ces «terrains» oriente vers des attentes distantes entre elles. L'existence du corps est différence, disparité. Telle est bien la vision première de ce champ dispersé. La pluralité s'accroît davantage encore, une fois pris en compte l'émiettement des sciences censées l'éclairer: du fonctionnement de l'organisme à l'imaginaire qu'il engendre, de la mécanique physique à la sensibilité qu'elle mobilise. Biologie, psychologie, anthropologie ou sociologie sont, à leur manière, et différemment, concernées par un tel champ. Ce qui n'implique pas leur convergence. Le corps ne constitue pas un «objet» unifié, faut-il le redire, au sens où l'entend la science classique. Une hétérogénéité s'impose ici, ajoutant à l'éloignement des pratiques celui des méthodes et des interprétations. L'approche elle-même multiplie les regards et les modes de connaissance: des repères les plus matériels aux savoirs les plus abstraits. Ce qui peut, tout au plus, suggérer une interdisciplinarité de démarche; alors que demeure plus difficile, sinon hasardeux, de suggérer une unité et convergence d'objet.

\section{Le projet}

C'est une orientation différente pourtant que j'ai recherchée et que je voudrais évoquer ici. Non pas pour postuler quelque unité dernière du corps. Mais pour rappeler l'existence de cohérences, au-delà des dispersions premières: évoquer des logiques unifiées traversant des champs différents. Nombre de rapprochements, à chaque époque précise, peuvent s'imposer. A commencer par les types d'influence «sculptant» les corps, créant au bout du compte des homogénéités. La faim ou le labeur bien sûr, mais aussi la culture, l'espace social. L'univers des cours princières ou royales par exemple rend convergents, au début de notre modernité, des gestes, des expressions, des valorisations de soi relevant de versants corporels censés être les plus différents. Un même principe, tout à fait inédit, de «délicatesse» peut ici trans- 
former «parallèlement» la vision de la nourriture, celle de l'apparence, celle des gestes quotidiens, celle de l'entretien de soi: le programme du courtisan est un programme global. Son corps y est, de part en part, «orienté». Il y est même dressé jusqu'à sa manière de «goûter», de marcher, de manger ou de jouer. Une même logique, celle de l'étiquette ou de la civilité, commande alors la diversité. Une référence unique traverse la pluralité. Un mot même répété à l'envie par Balthasar Castiglione dans son Livre du courtisan, en 1528 , me semblait décisif: celui de «légèreté», résumant à lui seul un principe d'apparence et de comportement. Alors qu'à un autre «bout» de l'échelle du temps, d'autres préoccupations, elles aussi «convergentes», l'emportent: l'attente d'efficacité, d'énergie, de rendement, pour ne citer qu'elles. La société industrielle, par exemple, suggère d'autres normes, d'autres modes d'évaluation, d'autres modes d'appréciation, d'autres types de regard, y compris sur l'apparence, la gestualité, la nourriture, la santé.

Cette recherche d'unité est bien la tentative de faire exister le corps comme un «objet» à lui tout seul, au-delà des diverses thématiques qui avaient déjà été largement explorées par les historiens: la nourriture, le vêtement, les maladies, les techniques, le nu dans l'art, les jeux, le sport. L'idéal que je tentais de poursuivre avec mes premières investigations était de faire exister «historiquement» le corps, comme Philippe Ariès avait fait exister le «sentiment», en l'occurrence ici l'amour pour l'enfant, dans son livre fondateur, L'enfant et la vie familiale sous l'ancien régime (1960). Cet idéal était aussi de retrouver ce que Lucien Febvre appelait très justement «l'homme en chair et en os», conviant, dès les années 1930, à en faire l'histoire. Une «unité corporelle» pouvait-elle exister, changeant «en totalité», suivant les normes des cultures et du temps?

\section{Représentations}

Impossible, par ailleurs et quoi qu'il en soit, d'ignorer la conscience. Impossible d'ignorer la représentation. L'histoire du corps ne saurait se limiter ici aux influences directes exercées par les matérialités. Le corps est aussi objet de «pensée». Ici, sans doute, pouvait se profiler quelque unité. Le corps est animé, dirigé, coordonné, ce qui peut renforcer des principes de convergence, voire d'homogénéité. Un modèle intériorisé de ce même corps existe: «système mental» liant les parties, orientant les mouvements, les perceptions, les états. Objet central même, ce modèle intérieur a lui aussi ses logiques, ses variations. 
Une notion, par exemple, m'avait toujours frappé, celle de «schéma corporel», ce «double intérieur», mis en évidence par les psychologues, ou plutôt les physio-psychologues, du début du $\mathrm{XX}^{\mathrm{e}}$ siècle, et depuis largement développé. Les définitions étaient abstraites, parfois pompeuses, mais convaincantes: «schéma de nos attitudes, des positions respectives des segments de nos membres, enfin une image du revêtement simple et élastique qui enveloppe notre corps». ${ }^{1}$ L'idée d'une représentation totale et physique de soi-même: «la sensation du corps propre considéré comme un tout dans le monde des phénomènes», ${ }^{2}$ disait même une définition plus austère. Impossible, sans ce repère, de situer la place des membres, leur orientation dynamique. Impossible, sans lui, d'ajuster les parties, ou, tout simplement, de bouger. Le schéma corporel se donnait en topographie virtuelle orchestrant attitudes et mouvement. ${ }^{3}$ Les psychologues multipliaient les indications: l'illusion de l'amputé par exemple dont la sensation durable du membre perdu confirmait l'existence d'une image intérieure constante et achevée, ou l'absence de cette même image chez le jeune enfant, par exemple, dont les dessins de bonhommes demeuraient longtemps sans jambes ni bras.

Cette image, non toujours consciente bien évidemment, me semblait remarquable. Les psychologues y voyaient une «condition de la conscience et de ses actes», ${ }^{4}$ une manière de polariser l'existence, l'installer entre les choses et les gens, orienter l'action. Ses «perturbations», du coup, s'avéraient redoutables. Une pathologie du «schéma corporel» existait, conduisant à des perceptions internes radicalement bouleversées. Et, pour dire brutalement, elle me fascinait. Julian de Ajuriaguerra et Henri Hécaen citaient, dans leur livre majeur, quelques cas étonnants. Telle cette patiente craignant en permanence de «tomber en morceaux», prétendant un jour avoir de «l'eau plein le corps», le lendemain avoir «le corps en feu», un autre jour avoir «le corps sale et noir», un autre encore avoir «les nerfs trop longs». Son «intérieur» jouait le chaos: sa «fesse droite se décollait», sa bouche «était dans son ventre», ses «gencives s'arrachaient». ${ }^{5}$ Dans certains cas encore le corps lui-même était jugé absent, entraînant une inéluctable perte de soi: «Je n'ai plus la notion de moi-même» ${ }^{6}$, disait une patiente impuissante à percevoir son corps. Ou, plus profondément encore: «Je cherche à penser, je ne peux

1 Thomas 1942, 17.

2 Klaus Conrad, «Das Körperschema, eine kritische Studie und der Versuch einer Revision», Zeitschrift für die gesamte Neurologie und Psychiatrie 147 (1933) 346-369, cité par Ajuriaguerra/Hécaen 1952, 51.

3 Alain Berthoz parle d'un «corps virtuel»: Berthoz 2003, 152.

4 Zazzo 1976, 18.

5 de Ajuriaguerra/Hécaen 1952, 272.

6 de Ajuriaguerra/Hécaen 1952, 279. 
me représenter». ${ }^{7}$ Autant de constats suraccentuant l'importance du thème. Une «fantasmagorie» très spéciale semblait ainsi pensable, sinon nommable. Le corps pouvait suggérer mille «images» internes, mille représentations faussement cohérentes ou structurées, toutes susceptibles d'influencer la réalité physique et sa vérité. D'où ces inévitables conséquences sur la manière de vivre et de s'éprouver.

\section{Modèles}

Une hypothèse se profilait transposant le thème du schéma corporel aux cultures et suggérant des variations curieuses ou notables avec le temps. Le livre de Judith Schlanger, Les métaphores de l'organisme (1971) était une source de suggestions. ${ }^{8}$ Elle multipliait les exemples de rapprochements et de raisonnements analogiques: des métaphores venues du climat à celles venues de l'industrie, les unes et les autres prétendant rendre compte, sur un mode tout imaginaire, des logiques du corps. Le texte de Canguilhem sur «Machine et organisme ${ }^{9} »$ me paraissait plus important encore.

Un seul exemple alors de ces «modèles»: une image du corps transmise par la médecine et la philosophie grecques domine les références corporelles occidentales quasiment jusqu'au XVIII ${ }^{\mathrm{e}}$ siècle. Cette image promeut une matière en son centre, les humeurs: ensemble de liquides censés constituer l'essence de l'organique. Ces liquides se voient, se touchent, se sentent, répondant à la logique la plus immédiate: l'assimilation de la vie à ce qui «coule». Ils émergent à la moindre effraction ou blessure, ils existent dans la transpiration, ils composent les déchets, confirmant l'amalgame entre fluides et vitalité. Plus encore, ces liquides s'effacent avec la vie, laissant desséchée la peau des malades ou celle des mourants. ${ }^{10}$ Autant dire que cette représentation centrale dans les pratiques anciennes commande les actes les plus différents en les unifiant: fréquenter un air dont la pureté assure celle des liquides, ajuster une nourriture facilitant cette pureté supposée, évacuer régulièrement par saignée, purger les liquides censés se corrompre ou s'accumuler, compenser par des assèchements divers l'effet de climats jugés trop humides, ou par des humidifications diverses l'effet de climats jugés trop secs. L'ensemble du comportement, au-delà de sa disparité, est dans ce cas orienté

7 Lhermitte 1939, 149.

8 Schlanger 1971.

9 Canguilhem 1965.

10 Voir, sur les humeurs, Andries 1994, en particulier le chapitre 3: «La médecine du corps et de l'âme». 
par une même vision du corps, jusqu'aux gestes les plus banals comme ceux de se coiffer, de dormir ou de s'habiller, ceux qui peuvent ici servir à une gestion ou à une évacuation voulues «heureuses» et calculées des humeurs.

Autant dire que ces repères n'auront plus de sens lorsque Diderot suggère à son d'Alembert endormi, en 1769 , un rêve de matière organique et dès lors un rêve de représentation du corps. Lui aussi s'attarde aux constituants présumés de cette matière. Il s'arrête en revanche non aux humeurs, mais aux «écheveaux» et aux «brins», aux «lacis» et aux «faisceaux», tous filaments vibratiles censés orchestrer un «système purement sensible». ${ }^{11}$ Le modèle du corps, dans ce cas, a définitivement basculé. L'«essentiel» du corps n'est plus ce qui «coule», il est ce qui, en toute priorité, entretient l'alerte et la stimulation. La référence lointaine semble devenue l'électricité plus que l'eau. L'électricité, parce que la séduction des représentations n'est pas étrangère aux transformations contemporaines du milieu: à celle des forces jugées opérantes, aux découvertes techniques du temps, à leur pourvoir de séduction imaginaire. D'où cette électricité brusquement «fascinante» dans la deuxième moitié du XIX ${ }^{\mathrm{e}}$ siècle, suggérant métaphores et principes d'explication, voire de rationalité. D'où encore l'inévitable changement dans l'«analyse» du mal, comme l'inévitable changement dans les pratiques d'entretien et de santé, voire même dans celles de l'éducation et de la normativité. La certitude de Bordeu par exemple, en 1775, elle aussi totalement datée: «Toutes les maladies ne sont à proprement parler que des maladies nerveuses.» ${ }^{12}$ C'est en terme d' «affermissement», voire même de durcissement, que commence alors à se définir la santé, comme la «formation» du corps. La «consolidation» des fibres l'emporte sur le geste d'évacuation.

Inutile d'envisager ici l'ensemble des modèles, même si se devinent déjà bien d'autres exemples: ceux du XIX ${ }^{\mathrm{e}}$ siècle, entre autres, avec leurs références à l'oxygène renforçateur, aux chimies, au rendement, à l'énergie. C'est le «moteur» qui devient alors central: la capacité à exploiter les ressources combustives, la bonne marche des poumons, l'équilibre énergétique des aliments, la puissance et le dosage du feu organique. La machine à feu et sa puissance de conviction l'emportent dans l'imagination «commune». Humeurs ou fibrilles perdent leur ancien sens, la santé, la bonne marche du corps, jusqu'à son apparence privilégiée, basculant vers de tout autres repères lorsque s'imposent ces indices caloriques, l'attention calculée aux mesures des entrées et des sorties, leur stabilité, leur efficacité. Alors que changent encore les modèles du $\mathrm{XX}^{\mathrm{e}}$ siècle avec leurs références aux hormones, aux vitamines, aux psychologies, aux équilibres nerveux. Les indices s'intério-

11 Diderot 1951 (1769), 904, 930.

12 de la Roche 1778, t. I, 13. 
risent, la santé se personnalise, l'idéal corporel se «spiritualise», orientant encore vers de nouveaux repères, de nouvelles pratiques, de nouvelles voies. Les exemples précédents suggèrent au moins l'existence de modèles du corps. Il faut retenir leur principe, leur effet de rupture, leurs successions possibles, leur rôle dans l'entretien d'un corps, son fonctionnement, son apparence, toujours divers autant que relativement unifiés.

Aucun doute, un inévitable commentaire doit accompagner ce parti pris $\mathrm{du}$ «modèle». Le mot par exemple ne saurait être entendu avec la précision que lui accordent les épistémologues. Il ne porte pas sur les mêmes repères. Son objet est aussi varié et étendu que particulier, visant prioritairement des pratiques, leur diversité, leur richesse, leur manque, quelquefois, d'explicitation. D'où la spécificité de la convergence recherchée. Elle a pour objet le savoir davantage que la science, par exemple. Elle est assimilable à une représentation dominante, plus qu'à quelque combinaison chiffrée, symbole des modèles savants. Elle peut entretenir de l'approximatif, du confus: repère flou et pourtant convaincant, générique et pourtant cohérent, ouvert et pourtant opérant. Cet objet ressemblerait plutôt à une «représentation sociale», telle que l'ont entendue les psychologues: «activité mentale orientée vers la pratique, servant de guide d'action concrète sur les hommes et les choses». ${ }^{13}$ Ce qui ne manque pas d'unité puisque cette pensée «vise la systématisation des savoirs pragmatiques». ${ }^{14}$ Elle les coordonne, les rassemble, leur donne conviction et densité.

L'enjeu pour la recherche historique devient alors, plus largement, de montrer comment, pour une même époque, s'est insensiblement imposée, d'un objet à un autre, d'un texte à un autre, d'une image à une autre, une représentation «identique» du corps: quelque «unité mentale» traversant la diversité de ses pratiques, de ses gestes, de ses attentes, en leur donnant cohérence et légitimité. Cette unité concernerait autant les repères de fonctionnement internes que ceux d'apparence externe, autant la mécanique que la sensibilité.

\section{Pratiques}

Reste, et il faut y insister, que l'investigation à laquelle je me livrais visait d'abord des pratiques plus que des théories. A commencer par mon premier travail sur le «redressement» du corps. C'est une profusion éminemment concrète qui me retenait: dispositifs, manières de faire, instruments, positions.

13 Jodelet 1984, 30-31.

14 Jodelet 1984, 30-31.

Gesnerus 70 (2013) 
Le redressement est un univers de gestes, dès les périodes les plus anciennes: un foisonnement de «faire» et d'actions. Mais un «faire» orienté. Les premières manipulations appliquées au nourrisson en étaient l'exemple: celles conseillées par les textes antiques, comme par les textes de l'univers classique. Un ensemble d'emprises précises: «À pleines mains on fera des frictions sur le dos pendant que le tronc sera maintenu par une traction afin de lui donner une forme convenable.» ${ }^{15}$ Les précautions se multiplient dans ces recommandations les plus anciennes, les traitements aussi, censés, par exemple, mieux différencier filles et garçons. Pour les filles, les inflexions vertébrales seraient quasiment à créer: «L'index et le médius appliqués au-dessus des fesses creusent une dépression gracieuse. ${ }^{16}$ Alors que le «droit» l'emporte pour les garçons. Les chairs se différencient selon les pressions exercées. Méthode identique, en revanche, pour renforcer les parties en les immobilisant: «Les déligations (applications méthodiques de bandages) journalières des membres rendent les nerfs plus forts.» ${ }^{17}$ Autant de gestes redresseurs perçus quasiment comme autant d'engendrements: ceux qu'un auteur italien du XVI ${ }^{\mathrm{e}}$ siècle n'hésite pas à intituler: «de faciendo infante» («fabriquer un enfant»). ${ }^{18}$ Pétrir, malaxer, re-former confinent au projet de faire entrer l'enfant dans les normes d'une communauté. Appliquer la main serait un rituel formateur. Autant de gestes inévitablement liés aussi à des représentations. Le corps des plus jeunes est ici fait d'une substance saturée de liquides, imprégnée d'eau, si humide même que ses os demeureraient longtemps malléables et «orientables». D'où cette idée simplissime selon laquelle des bandes textiles fermement appliquées poursuivent le travail correcteur. Redresser serait d'abord «pétrir».

Ce thème des pratiques est particulier, surtout lorsqu'il vise les pratiques collectives. Sa recherche joue avec le discours et ne s'en satisfait pas. Elle l'utilise bien sûr, tout en recourant d'abord à sa diversité. La littérature, par exemple, est tout aussi intéressante que la médecine pour indiquer quelles sont les pratiques de bain au XVIII ${ }^{\text {e }}$ siècle, alors que s'impose un nouveau goût pour l'eau et que se redéfinit la mondanité. Le texte de jurisprudence, par ailleurs, est tout aussi intéressant que celui du médecin légiste pour indiquer la gravité accordée à une blessure en cas d'agression ou de viol, lorsque grandissent au XVIII ${ }^{\mathrm{e}}$ siècle les principes de sensibilité. L'image enfin est tout aussi, sinon plus, importante que le texte, pour indiquer la valeur des tenues physiques et leur changement entre le XVIII ${ }^{\mathrm{e}}$ et le XIX ${ }^{\mathrm{e}}$ siècle.

15 Soranus d'Éphèse 1895, 1.

16 Soranus d'Éphèse 1895, 115-116.

17 Guillemeau 1621, 396.

18 Ferrarius 1577, 49. 
Un seul exemple: le profil physique, largement recomposé au début du XIX siècle. Le modèle corporel de l'aristocratie, en France, y est subverti. Celui de l'homme surtout:non plus l'avancée du ventre, par exemple, épaules repoussées en arrière évoquant quelque hauteur «noble», mais le buste droit, densifié, ceinture retenue et comprimée, évoquant quelque détermination «bourgeoise». Non plus l'honneur dans un profil arqué, mais la vigueur dans un tronc renforcé: moins d'arrogance sans doute, plus d'efficacité. L'apparence physique est métamorphosée. Tenue ouvertement active, l'ampleur du buste se déploie en signe de force et de capacité. Tout a changé avec ce début du XIX ${ }^{\mathrm{e}}$ siècle, tout sépare la redingote nouvelle, par exemple, du pourpoint traditionnel: les aplombs, les lignes, la verticalité. Les épaules s'étoffent en revers démesurés. La poitrine surplombe un ventre étranglé. Ce qui bouleverse l'enseignement du modèle physique: les préceptes édictés dans les salles de danse, dans les salles d'armes, dans les gymnases, et même dans les salles de classe. Ce qui donne à la poitrine une importance sans égale jusque-là, conduisant à inventer mille mouvements pour mieux la développer. Ce qui confirme aussi, à sa manière, combien étudier l'attitude peut conduire à mieux étudier un univers culturel et mental, son épaisseur, ses spécificités, ses changements. Autant dire que seules les images témoignent de ce changement. Seules elles «montrent» comme elles confirment.

Impossible alors de ne pas conclure sur un exemple: celui de la convergence de représentations mêlant le fonctionnement du corps et son apparence, l'imaginaire du dedans et l'imaginaire du dehors. La vision inédite de l'énergie au début du XIX ${ }^{\mathrm{e}}$ siècle le montre. L'image de la vie transformée, image partagée par le plus grand nombre: cette vie brusquement perçue comme dépense énergétique et principe de rendement, le poumon devenu lieu de combustion. Autant de thèmes qui font basculer de part en part l'allure et la tenue physiques. La poitrine se tend sous le gilet des hommes comme elle s'accroît sous le corsage des femmes au début du XIX ${ }^{\mathrm{e}}$ siècle. La mode vestimentaire peut même multiplier les rembourrages pour mieux souligner ce déploiement. L'attention à un air «brûleur» réoriente encore les pratiques sanitaires, sensibles aux effets «revigorants» prêtés à l'oxygène, comme elle réoriente les pratiques alimentaires, sensibles à la force combustive des denrées, ou comme elle réoriente l'évaluation du travail, sensible aux ressources combustives de l'ouvrier. Une même vision de l'énergie traverse des pratiques différentes en ce début du XIX ${ }^{\mathrm{e}}$ siècle. Une même représentation du corps relie l'esthétique, le labeur, l'aliment, la santé. Il n'est jusqu'à l'éducation qui, au même moment, se doit de développer les poitrines enfantines pour mieux les «protéger». 
Un raisonnement identique peut être tenu pour d'autres modèles de corps bien sûr. La fascination exercée aujourd'hui par les repères «informationnels» par exemple, l'importance donnée à l'écoute des sens, le rapprochement entre les dispositifs organiques et les dispositifs informatiques. Nouvelles «synthèses», ici encore: les valeurs physiques pensées davantage selon les ressources réactives, par exemple, les silhouettes pensées davantage selon les qualités d'auto-contrôle, le corps travailleur pensé davantage selon ses capacités de réponse aux signaux, sa résistance aux charges mentales, le corps nourri pensé davantage selon les exigences nerveuses, l'accroissement des lucidités.

Une des originalités de l'histoire du corps est bien d'orienter vers des représentations «unifiantes». Elle éclaire une époque, elle souligne sa culture, autant qu'elle en souligne la possible«homogénéité».

\section{Bibliographie}

Andries, Lise, Le Grand Livre des Secrets. Le colportage en France aux $17^{e}$ et $18^{e}$ siècles (Paris 1994)

Berthoz, Alain, La décision (Paris 2003)

Canguilhem, Georges, «Machine et organisme», in: Georges Canguilhem, La connaissance et la vie (Paris 1965) 101-127

de Ajuriaguerra, Julian/Henri Hecaen, Méconnaissances et hallucinations corporelles, intégration et désintégration de la somatognosie (Paris 1952)

de la Roche, Daniel, Analyse des fonctions du système nerveux (Genève 1778)

Diderot, Denis, Le rêve de d'Alembert (1769), in: Euvres (Paris 1951)

Ferrarius, Omnibonus, De arte medica infantium, libri quatuor, quorum duo priores de tuenda eorum sanitate, posteriores de curandis morbis agunt (Brescia 1577)

Guillemeau, Jacques, De la grossesse et accouchement des femmes; du gouvernement de celles-ci et moyen de survenir aux accidents qui leur arrivent, ensemble de la nourriture des enfans (Paris 1621)

Jodelet, Denise, «Réflexions sur le traitement de la notion de représentation sociale en psychologie sociale», in: Bernard Schiele/Claire Bélisle (éds), Les Représentations. Numéro spécial de la revue Communication Information 6 (1984) 15-41

Lhermitte, Jean, L'image de notre corps (Paris 1939)

Schlanger, Judith, Les métaphores de l'organisme (Paris 1971)

Soranus d'Ephèse, Traité des maladies des femmes, traduit et annoté par François Joseph Hergott (Nancy 1895)

Thomas, André, «L'image de mon corps», Revue neurologique 74, 1942, 1-19

Zazzo, René, Conduites et conscience (Neuchâtel/Paris 1976) 